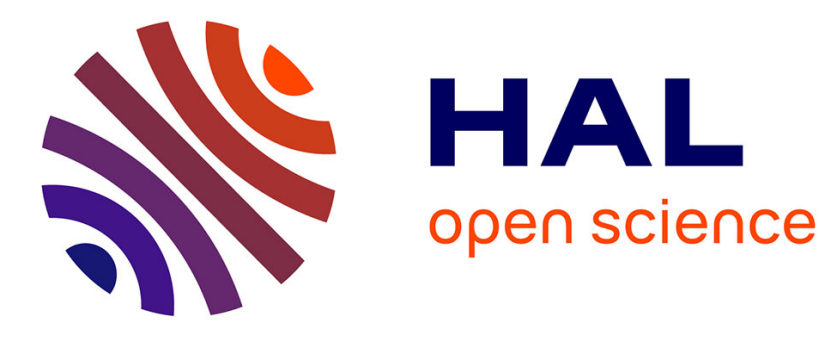

\title{
Morphological Floodings and Optimal Cuts in Hierarchies
}

Jean Cousty, Laurent Najman

\section{To cite this version:}

Jean Cousty, Laurent Najman. Morphological Floodings and Optimal Cuts in Hierarchies. International Conference on Image Processing (ICIP), Oct 2014, Paris, France. 10.1109/ICIP.2014.7025905 . hal-01082353

\section{HAL Id: hal-01082353 \\ https://hal.science/hal-01082353}

Submitted on 13 Nov 2014

HAL is a multi-disciplinary open access archive for the deposit and dissemination of scientific research documents, whether they are published or not. The documents may come from teaching and research institutions in France or abroad, or from public or private research centers.
L'archive ouverte pluridisciplinaire HAL, est destinée au dépôt et à la diffusion de documents scientifiques de niveau recherche, publiés ou non, émanant des établissements d'enseignement et de recherche français ou étrangers, des laboratoires publics ou privés. 


\title{
MORPHOLOGICAL FLOODINGS AND OPTIMAL CUTS IN HIERARCHIES
}

\author{
Jean Cousty and Laurent Najman \\ Université Paris-Est, LIGM, A3SI, ESIEE Paris
}

\begin{abstract}
The non-horizontal cuts of a hierarchy and the floodings of an image are well-established tools for image segmenting and filtering respectively. We present definitions of non-horizontal cuts and of floodings in the same framework of hierarchies of partitions. We show that, given a hierarchy, there is a one-to-one correspondence between the non-horizontal cuts and the floodings. This opens the door to optimal image filtering based on non-horizontal cuts and, conversely, to nonhorizontal cuts obtained by morphological floodings, or more generally by connected filterings.
\end{abstract}

Index Terms - hierarchy, non-horizontal cut, flooding

\section{INTRODUCTION}

V. Caselles, to whom this paper is dedicated, has made some very important and remarkable contributions to image processing $[1,2]$. He was first of all, a mathematician, and was eager to demonstrate that great mathematical ideas can lead to great algorithms for solving real-world problems. He was interested in hierarchical/tree-like structures (notably trees of shapes [3] and hierarchical segmentations [4]), realizing both their theoretical interest and their importance for practical applications. In fact, in the framework of [5, 6] where segmentations can be seen as partial partitions, trees of shapes are hierarchies of segmentations. This paper deals with hierarchical segmentations, and show some theoretical links between different approaches (and seemingly unrelated) existing in the literature, namely non-horizontal cuts [7] and morphological floodings [8]. While the results exposed here are primarily of a theoretical nature, we believe that these links pave the way for some novel practical image analysis tools.

Many image segmentation methods look for a partition of the set of image pixels such that each region of the partition corresponds to an object of interest in the image. Hierarchical segmentation methods, instead of providing a unique partition, produce a sequence of nested partitions at different scales, enabling to describe an object of interest as a grouping of several objects of interest that appear at lower scales.

This work received funding from the Agence Nationale de la Recherche, contract ANR-2010-BLAN-0205-03. through "Programme d'Investissements d'Avenir" (LabEx BEZOUT - ANR-10-LABX-58)
Two different approaches for selecting a partition from a hierarchy exist in the literature. The first one deals with cut of the hierarchy, which amounts to selecting various regions in the hierarchy to obtain a partition. L. Guigues et al. [7] were the first to explore the notion of non-horizontal cut, meaning that the regions are selected at several levels in the hierarchy. The choice of the "correct" regions is made by optimizing an energy functional (see [9] for a recent extension and [10] for earlier versions of similar ideas). In a recent work, V. Caselles et al. [4] explore the capabilities of the a contrario approach for selecting an optimal partition from the hierarchy.

A different approach, called flooding, is classical in mathematical morphology. It consists in processing the image function itself. Intuitively, a flooding fills some catchment basins. The flooding filtering $[8,11,12]$ is often paired with a watershed process in order obtain an image segmentation, especially to overcome the oversegmentation problem: a watershed applied on a flooding-filtered image provides less regions. By reiterating the flooding and the watershed, we thus obtain coarser and coarser partitions, and finally a whole hierarchy of partitions. Formally, the floodings of an image can be characterized $[13,14]$ from a hierarchical representation of the image called min-tree [15].

In this paper, we present definitions of non-horizontal cuts and of floodings in the same framework and we show that, given a hierarchy, there is a one-to-one correspondence between non-horizontal cuts and floodings. This opens the door to optimal image filtering based on non-horizontal cuts and, conversely, to non-horizontal cuts obtained by morphological floodings, or more generally by connected filterings. Previous works such as [16] allow the processing of hierarchies of connected partitions [17]. In this paper, the connectedness hypothesis is relaxed, i.e., the classes of the partitions are no longer required to be connected.

\section{HIERARCHIES OF PARTITIONS}

In this section, we recall some basic definitions for handling partitions and hierarchies of partitions.

A partition of a set $V$ is a set $\mathbf{P}$ of nonempty disjoint subsets of $V$ whose union is $V$ (i.e., $\forall X, Y \in \mathbf{P}, X \cap Y=\emptyset$ if $X \neq Y$ and $\cup\{X \in \mathbf{P}\}=V$ ). Any element of a partition $\mathbf{P}$ of $V$ is called a region (or a class) of $\mathbf{P}$. If $x$ is an element of $V$, there is a unique region of $\mathbf{P}$ that contains $x$; this 

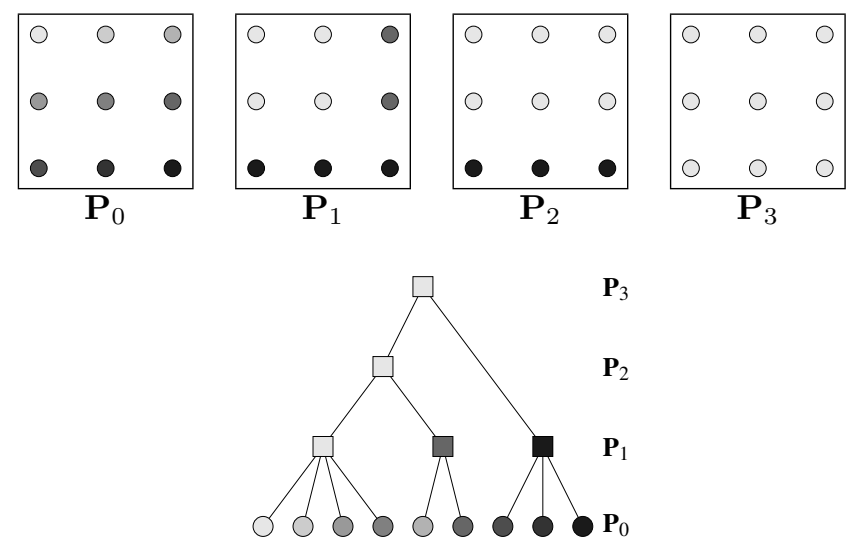

$\mathcal{H}$

Fig. 1. Illustration of a hierarchy $\mathcal{H}=\left(\mathbf{P}_{0}, \mathbf{P}_{1}, \mathbf{P}_{2}, \mathbf{P}_{3}\right)$. For each partition, each region is represented by a gray-level and two dots with the same gray level belong to the same region. The last subfigure represents the hierarchy as a tree, called a dendrogram, where the inclusion relation between the regions of the successive partitions is represented by line segments; when a same region appears in two successive partitions of the hierarchy, this region is only represented at the lowest level.

unique class is denoted by $\mathbf{P}_{x}$. Given two partitions $\mathbf{P}$ and $\mathbf{P}^{\prime}$ of a set $V$, we say that $\mathbf{P}^{\prime}$ is a refinement of $\mathbf{P}$ if any region of $\mathbf{P}^{\prime}$ is included in a region of $\mathbf{P}$. A hierarchy $($ on $V$ ) is a sequence $\mathcal{H}=\left(\mathbf{P}_{0}, \ldots, \mathbf{P}_{\ell}\right)$ of indexed partitions of $V$ such that $\mathbf{P}_{i-1}$ is a refinement of $\mathbf{P}_{i}$, for any $i \in\{1, \ldots, \ell\}$. A hierarchy $\mathcal{H}=\left(\mathbf{P}_{0}, \ldots, \mathbf{P}_{\ell}\right)$ is complete if $\mathbf{P}_{\ell}=\{V\}$ and $\mathbf{P}_{0}$ contains every singleton of $V$ (i.e., $\mathbf{P}_{0}=\{\{x\} \mid x \in V\}$ ). All hierarchies considered in this article are complete and therefore the term complete is omitted.

Fig. 1 graphically represents a hierarchy $\mathcal{H}=\left(\mathbf{P}_{0}, \mathbf{P}_{1}\right.$, $\mathbf{P}_{2}, \mathbf{P}_{3}$ ) on a rectangular subset $V$ of $\mathbb{Z}^{2}$ made of 9 dots. For instance, it can be seen that $P_{1}$ is a refinement of $P_{2}$ since any region of $P_{1}$ is included in a region of $P_{2}$. It can also be seen that the hierarchy is complete since $P_{0}$ is made of singletons and $P_{3}$ is made of a single region that contains all elements.

\section{NON-HORIZONTAL CUTS}

Let $\mathcal{H}=\left(\mathbf{P}_{0}, \ldots, \mathbf{P}_{\ell}\right)$ be a hierarchy on $V$. A subset $A$ of $V$ is a region of $\mathcal{H}$ if there exists $i$ in $\{0, \ldots \ell\}$ such that $A$ is a region of the partition $\mathbf{P}_{i}$. We denote by $\mathbf{R}_{\mathcal{H}}$ the set of all regions of $\mathcal{H}: \mathbf{R}_{\mathcal{H}}=\left\{A \in \mathbf{P}_{i} \mid i \in\{0, \ldots, \ell\}\right\}$.

Definition 1 (non-horizontal cut) Let $\mathcal{H}$ be a hierarchy. A partition $\mathbf{P}$ of $V$ that only contains regions of $\mathcal{H}$ (i.e., $\mathbf{P} \subseteq$ $\mathbf{R}_{\mathcal{H}}$ ) is called a (non-horizontal) cut of $\mathcal{H}$.

For instance, a cut of the hierarchy $\mathcal{H}$ depicted in Fig. 1 is depicted in Figs. 2(a) and (b). In Fig. 2(b), the cut is made of the regions represented immediately below the bold line.

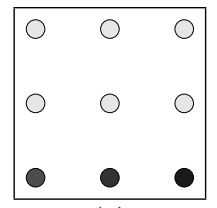

(a)

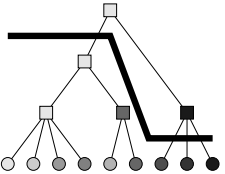

(b)

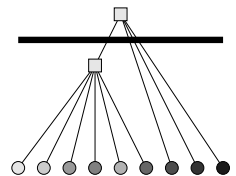

(c)
Fig. 2. Illustration of a non horizontal cut in a hierarchy and of the pruning by this cut. (a) A non horizontal cut $\mathbf{P}$ of the hierarchy $\mathcal{H}$ depicted in Fig. 1; (b) a representation of this cut (bold line) on the dendrogram of the hierarchy; (c) the dendrogram of the hierarchy $\mathcal{H}_{\mathbf{P}}$ which is the pruning of $\mathcal{H}$ by $\mathbf{P}$.

We are now going to see that any cut of a hierarchy $\mathcal{H}$ allows the transform of the hierarchy $\mathcal{H}$ into a simpler hierarchy called the pruning of $\mathcal{H}$ by the given cut. We first define the notion of pruning for partitions. Then the pruning of a hierarchy is defined as the pruning of all partitions in the hierarchy.

We say that a subset $A$ of $V$ is trivial if it is a singleton, i.e., if there exists $x$ in $V$ such that $A=\{x\}$. If $\mathbf{P}$ is a partition of $V$, we denote by $\mathbf{P}^{-}$the set of all elements of $\mathbf{P}$ that are trivial and by $\mathbf{P}^{+}$the set $\mathbf{P} \backslash \mathbf{P}^{-}$.

In general, the sets $\mathbf{P}^{+}$and $\mathbf{P}^{-}$are not partitions of $V$, but they are always partitions of a subset of $V$, hence they are partial partitions of $V[5,6]$.

Let $\mathbf{P}$ and $\mathbf{Q}$ be two partitions of $V$. The pruning of $\mathbf{P}$ by $\mathbf{Q}$ is the set $\mathbf{P}^{\prime}$ containing any region of $\mathbf{P}$ that includes a non trivial region of $\mathbf{Q}$ and any singleton included in a region of $\mathbf{P}$ which does not include any non-trivial region of $\mathbf{Q}: \mathbf{P}^{\prime}=$ $\left\{A \in \mathbf{P} \mid \exists B \in \mathbf{Q}^{+}, B \subseteq A\right\} \cup\left\{\{x\} \mid \forall B \in \mathbf{Q}^{+}, B \backslash[\mathbf{P}]_{x} \neq \emptyset\right\}$. In other words, the pruning of $\mathbf{P}$ by $\mathbf{Q}$ is obtained from $\mathbf{P}$ by keeping the regions which includes a non-trivial region of $\mathbf{Q}$ and by replacing the other regions of $\mathbf{P}$ by their singletons.

For instance, the pruning of the partition $\mathbf{P}_{0}$ (resp. $\mathbf{P}_{1}, \mathbf{P}_{2}$ and $\mathbf{P}_{3}$ ) shown in Fig. 1(a) (resp. (b), (c), and (d)) by the partition $\mathbf{P}$ of Fig. 2(a) is $\mathbf{P}_{0}$ (resp. $\mathbf{P}_{\mathbf{0}}, \mathbf{P}$ and $\mathbf{P}_{3}$ ).

In the context of partial partitions and hierarchies of partial partitions, an alternative definition for pruning would consist of removing some of the regions of the partition instead of replacing them by singletons. But in the context of this article, which studies hierarchies of partitions, we are interested in prunings that lead to partitions. Indeed, from the very definition of a pruning given in the previous paragraph, it can be seen that the pruning of a partition by another one is always a partition.

Let $\mathcal{H}=\left(\mathbf{P}_{0}, \ldots, \mathbf{P}_{\ell}\right)$ be a hierarchy and let $\mathbf{P}$ be a cut of $\mathcal{H}$. We call pruning of $\mathcal{H}$ by $\mathbf{P}$ the sequence of partitions $\mathcal{H}_{\mathbf{P}}=\left(\mathbf{P}_{0}^{\prime}, \ldots, \mathbf{P}_{\ell}^{\prime}\right)$ such that, for any $i$ in $\{0, \ldots, \ell\}$, the partition $\mathbf{P}_{i}^{\prime}$ is the pruning of $\mathbf{P}_{i}$ by $\mathbf{P}$.

For instance, Fig. $2 c$ depicts as a dendrogram the pruning of the hierarchy $\mathcal{H}$ of Fig. 1 by the cut represented in Figs. 2(a) and (b). 


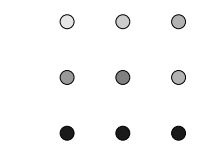

(a)

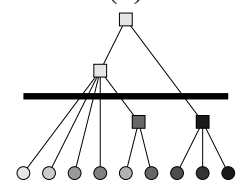

(c) $0_{x} \circ \circ$

$\circ \circ y_{\circ}$

$\circ \circ \circ$

(b)

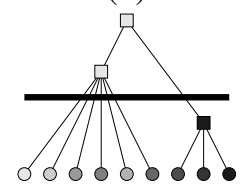

(d)
Fig. 3. Illustration of floodings. The partition depicted in (a) is the flooding of the partition $\mathbf{P}_{1}$ depicted in Fig. 1 by the set $A$ made of the vertices which are bold circled in (b). The dendrogram depicted in (c) (resp. (d)) represents the Mflooding $\mathcal{H}^{\prime}$ (resp. $\mathcal{H}^{\prime \prime}$ ) of the hierarchy $\mathcal{H}$ (resp. $\mathcal{H}^{\prime}$ ) by the point $x$ (resp. $y$ ), where $\mathcal{H}$ is the hierarchy depicted in Fig. 1 and where $x$ and $y$ are the points shown in (b). The cuts induced by these hierarchies $\mathcal{H}^{\prime}$ and $\mathcal{H}$ are superimposed in bold to the dendrograms.

As a direct consequence of the definition of prunings and cuts, we deduce the following property, which states that cuts can be used to transform a hierarchy into another hierarchy.

Property 2 Let $\mathcal{H}$ be a hierarchy and let $\mathbf{P}$ be a cut of $\mathcal{H}$. The pruning $\mathcal{H}_{\mathbf{P}}$ of $\mathcal{H}$ by $\mathbf{P}$ is a hierarchy.

\section{FLOODINGS AND NON-HORIZONTAL CUTS}

We are now going to study operators called floodings acting on hierarchies. These operators can also be extended to arbitrary maps. In the literature these operators are studied since the 90's (often without a formal definition) in the context of watershed segmentation and connected image filtering where they are sometimes called closing by reconstruction or swamping [8].

We start by defining the flooding of a partition and then we extend this definition to a hierarchy by considering floodings of all partitions of the hierarchy.

Let $\mathbf{P}$ be a partition of $V$ and let $A \subseteq V$. The flooding of $\mathbf{P}$ by $A$, denoted by flood $_{A}(\mathbf{P})$, is $\mathbf{P}$ if $A$ is not a region of $\mathbf{P}$, and otherwise, if $A$ is a region of $\mathbf{P}, \operatorname{flood}_{A}(\mathbf{P})$ contains any region of $\mathbf{P}$ distinct from $A$ and any singleton of $A$ :

$$
\begin{array}{lll}
\operatorname{flood}_{A}(\mathbf{P}) & =\mathbf{P} & \text { if } A \notin \mathbf{P} ; \\
\operatorname{flood}_{A}(\mathbf{P}) & =(\mathbf{P} \backslash A) \cup\{\{x\} \mid x \in A\} & \text { if } A \in \mathbf{P} .
\end{array}
$$

Fig. 3(a) shows an example of a flooding of a partition by a set of points.

From the definition, it can be seen that any flooding of a partition is a partition.

Let $\mathcal{H}=\left(\mathbf{P}_{0}, \ldots, \mathbf{P}_{\ell}\right)$ be a hierarchy, let $k$ in $\{1, \ldots, \ell\}$, and let $M$ be a non trivial region of $\mathbf{P}_{k}$. We say that $M$ is a (non-trivial) minimum of $\mathcal{H}$ if any subset of $M$ in $\mathbf{P}_{k-1}$ is trivial. We denote by $\mathbf{M}^{\mathcal{H}}$ the set of all minima of $\mathcal{H}$. We also denote by $M^{\mathcal{H}}$ the set of all elements of $V$ that belong to a minimum of $\mathcal{H}$. If $x$ is an element in $M^{\mathcal{H}}$, then we denote by $\mathbf{M}_{x}^{\mathcal{H}}$ the minimum of $\mathcal{H}$ that contains $x$. Observe that $\mathbf{M}^{\mathcal{H}}$ is not, in general, a partition of $V$. Indeed, in general, there exists some points of $V$ that do not belong to any minimum of $\mathcal{H}$. For instance, if we consider the hierarchy $\mathcal{H}$ of Fig. 3(c), the four leftmost dots do not belong to any minimum of $\mathcal{H}: \mathcal{H}$ contains only two minima that correspond to the two darkest squares.

In the following, if $\mathcal{H}$ is a hierarchy, we denote by $D^{\mathcal{H}}$ the set of all points in $V$ that do not belong to any minimum of $\mathcal{H}$, i.e. $D^{\mathcal{H}}=V \backslash M^{\mathcal{H}}$. We also denote by $\mathbf{D}^{\mathcal{H}}$ the set of all singletons included in $D^{\mathcal{H}}$.

Let $\mathcal{H}=\left(\mathbf{P}_{0}, \ldots, \mathbf{P}_{\ell}\right)$ be a hierarchy and let $x \in V$. If $x$ belongs to $M^{\mathcal{H}}$, the minimum-flooding, or simply $M$-flooding, of $\mathcal{H}$ by $x$ is the sequence of partitions $\mathcal{H}^{\prime}=\left(\mathbf{P}_{0}^{\prime}, \ldots, \mathbf{P}_{\ell}^{\prime}\right)$ such that, for any $i$ in $\{1, \ldots \ell\}$, the partition $\mathbf{P}_{i}^{\prime}$ is the flooding of $\mathbf{P}_{i}$ by $\mathbf{M}_{x}^{\mathcal{H}}$, i.e., $\mathbf{P}_{i}^{\prime}=\operatorname{flood}_{\mathbf{M}_{x}^{\mathcal{H}}}(\mathbf{P})$. If $x$ belongs to $D_{\mathcal{H}}$, the $M$-flooding of $\mathcal{H}$ by $x$ is $\mathcal{H}$ itself.

It can be seen that the M-flooding of $\mathcal{H}$ by $x$ is a hierarchy. This hierarchy is obtained by replacing $\mathbf{M}_{x}^{\mathcal{H}}$ by its singletons.

For instance, the hierarchy $\mathcal{H}^{\prime}$ in Fig. $3 \mathrm{c}$ is the M-flooding of the hierarchy $\mathcal{H}$ in Fig. 1 by the point $x$ shown in Fig. $3 \mathrm{~b}$ The hierarchy $\mathcal{H}^{\prime \prime}$ (Fig. 3d) is the M-flooding of $\mathcal{H}^{\prime}$ by $y$.

Definition 3 (flooding) Let $\mathcal{H}$ and $\mathcal{H}^{\prime}$ be two hierarchies. If there exists $x$ in $V$ such that $\mathcal{H}^{\prime}$ is the M-flooding of $\mathcal{H}$ by $x$, then we say that $\mathcal{H}^{\prime}$ is an elementary flooding of $\mathcal{H}$. We say that $\mathcal{H}^{\prime}$ is a flooding of $\mathcal{H}$ if there exists a sequence $\left(\mathcal{H}_{0}, \ldots, \mathcal{H}_{n}\right)$ of hierarchies such that $\mathcal{H}_{0}=\mathcal{H}, \mathcal{H}_{n}=$ $\mathcal{H}^{\prime}$, and $\mathcal{H}_{i}$ is an elementary flooding of $\mathcal{H}_{i-1}$, for any $i$ in $\{1, \ldots, n\}$.

For instance, the hierarchies $\mathcal{H}^{\prime}$ and $\mathcal{H}^{\prime \prime}$ of Figs. 3(c) and (d)are two floodings of the hierarchy $\mathcal{H}$ of Fig. 1.

Let us now state the main result of this section that establishes the correspondence between floodings and cuts.

If $\mathcal{H}$ is a hierarchy, we call partition induced by $\mathcal{H}$, the partition, denoted by $\boldsymbol{\Gamma}(\mathcal{H})$, that contains any minimum of $\mathcal{H}$ and any singleton on $V$ which is not included in a minimum of $\mathcal{H}: \mathbf{\Gamma}(\mathcal{H})=\mathbf{M}^{\mathcal{H}} \cup \mathbf{D}^{\mathcal{H}}$.

Property 4 Let $\mathcal{H}$ be a hierarchy. The partition induced by any flooding of $\mathcal{H}$ is a cut of $\mathcal{H}$.

Theorem 5 Let $\mathcal{H}$ be a hierarchy on $V$. The map $\Gamma$ is a oneto-one correspondence between the floodings of $\mathcal{H}$ and the non-trivial cuts of $\mathcal{H}$. The inverse $\boldsymbol{\Gamma}^{-1}$ of $\boldsymbol{\Gamma}$ maps to any cut $\mathbf{P}$ of $\mathcal{H}$ the pruning of $\mathcal{H}$ by $\mathbf{P}: \boldsymbol{\Gamma}^{-1}(P)=\mathcal{H}_{\mathbf{P}}$. More precisely, the following statements hold true:

- for any flooding $\mathcal{H}^{\prime}$ of $\mathcal{H}, \boldsymbol{\Gamma}\left(\mathcal{H}^{\prime}\right)$ is a cut of $\mathcal{H}$ such that the pruning of $\mathcal{H}$ by the cut $\boldsymbol{\Gamma}\left(\mathcal{H}^{\prime}\right)$ is precisely $\mathcal{H}^{\prime}$; and - for any non-trivial cut $\mathbf{P}$ of $\mathcal{H}$, the pruning $\mathcal{H}_{\mathbf{P}}$ of $\mathcal{H}$ by $\mathbf{P}$ is a flooding of $\mathcal{H}$ such that the partition induced by $\mathcal{H}_{\mathbf{P}}$ is $\mathbf{P}$. 


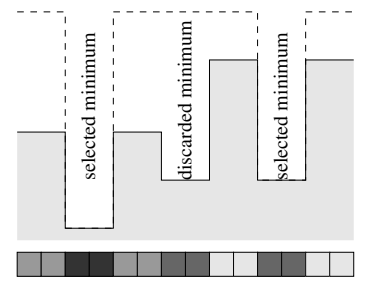

(a)

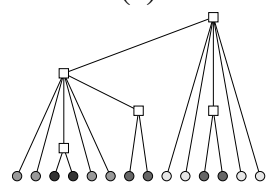

(c)

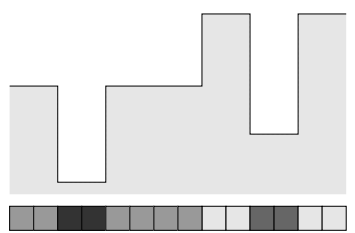

(b)

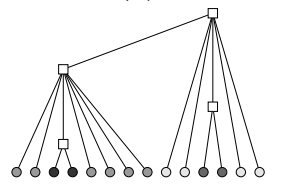

(d)
Fig. 4. Flooding of an image. (a) A greyscale 1D image and its topographical interpretation; (b) a geodesic reconstruction of (a) from the selected minima; (c) the min-tree of (a); (d) an elementary flooding of (c) which is the min-tree of (b).

\section{ILLUSTRATIONS AND CONCLUSION}

Geodesic reconstruction is a basis for connected filtering based on morphological attributes [15]. Any greyscale image can be equivalently represented by its min-tree [15] (i.e. the connected components inclusion hierarchy of the lower image thresholds). Let $\mathcal{M}$ be the set of regional minima of an image $I$, and let $X$ be one of these minima. The geodesic reconstruction of $I$ from $\mathcal{M} \backslash\{X\}$ as defined in [8] is precisely the image corresponding to the flooding by $x \in X$ of the min-tree of the image $I$ (see, e.g., Fig. 4). In the framework presented in this article, the image resolution must be doubled before computing the hierarchy so that the considered image do not contain trivial minimum that cannot be "flooded".

Note that, as illustrated in Fig. 5, the very same flooding process can be applied to any hierarchy representing an image (e.g. the quasi flat-zones of a greyscale image). This can be useful for obtaining non-horizontal cuts in a hierarchy based on floodings, hence based on morphological attributes of the regions in the hierarchy. Fig. 6 illustrates a flooding process on the constrained-connectivity hierarchy [19] of a real image Fig. 6a. It can be noted that this hierarchy Fig. $6 \mathrm{~b}$ exhibit numerous small zones (transition zones [20]) at a very high level. Hence a horizontal cut cannot remove those small zones. The flooding process with a volume attribute leads to non-horizontal cuts (Figs. 6c,d) removing those small zones.

Conversely, a last consequence of Th. 5 is that connected filters can benefit from optimal non-horizontal cuts such as [4] (Number of False Alarms) or [7, 9] (Mumford-Shah energies). In Figs. 6(e) and (f), a flooding is obtained as a pruning by an optimal (for a Mumford-Shah energy) non-horizontal cut of the component tree of the image. Future works include the assessment of the practical benefits of floodings by nonhorizontal cuts and of non-horizontal cuts by floodings.

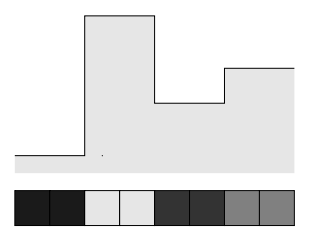

(a)

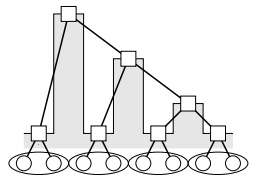

(c)

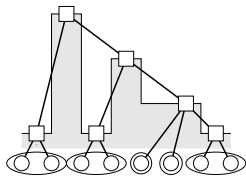

(d)

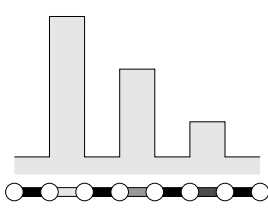

(b)

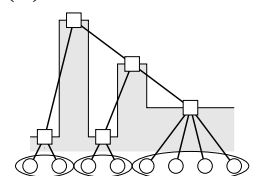

(e)
Fig. 5. Flooding of the quasi-flat zones hierarchy of a greyscale image. (a) A greyscale 1D image; (b) the gradient magnitude of (a) depicted as an edge-weighted graph; (c) the quasi flat-zones hierarchy of (a) which is also the min-tree of (b) (see [18]); (d) an elementary flooding of (c); (e) a flooding of (c), which is also an elementary flooding of (d). Note that the cut induced by each hierarchy is the partition given by the ellipsoid at the bottom of subfigures (c), (d) and (e).

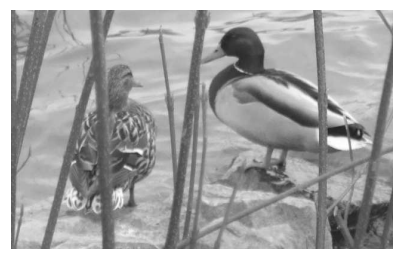

(a)

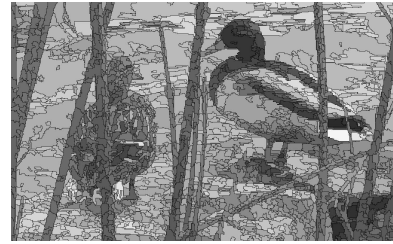

(c)

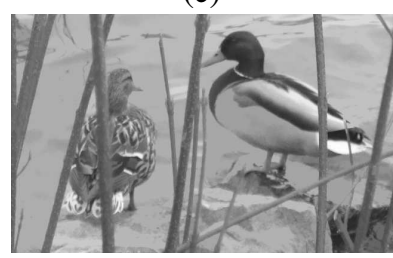

(e)

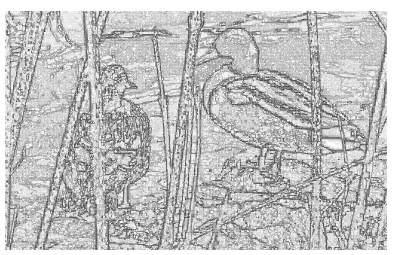

(b)

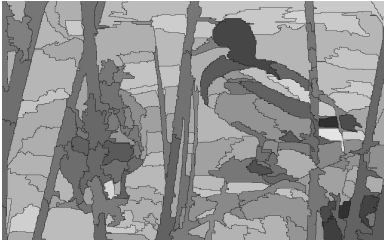

(d)

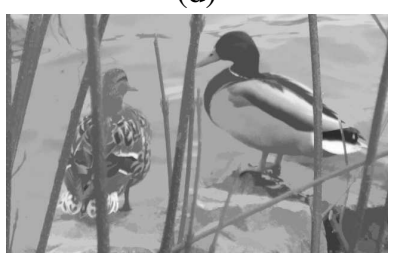

(f)
Fig. 6. (a-d) Cut by flooding in the constrained-connectivity hierarchy [19] of a greyscale image (a). (b) The saliency map $[21,16]$ of this hierarchy. (c,d) Two non-horizontal cuts by flooding with a volume-based attribute. (e,f) An opening by reconstruction (dual of a flooding) and an alternate-filter of (a) obtained from an optimal cut according to Mumford-Shah energy. 


\section{REFERENCES}

[1] Vicent Caselles, Ron Kimmel, and Guillermo Sapiro, "Geodesic active contours," International journal of computer vision, vol. 22, no. 1, pp. 61-79, 1997.

[2] Vicent Caselles, Francine Catté, Tomeu Coll, and Françoise Dibos, "A geometric model for active contours in image processing," Numerische mathematik, vol. 66, no. 1, pp. 1-31, 1993.

[3] Vicent Caselles and Pascal Monasse, Geometric description of images as topographic maps, Springer Publishing Company, Incorporated, 2009.

[4] Juan Cardelino, Vicent Caselles, Marcelo Bertalmio, and Gregory Randall, "A contrario selection of optimal partitions for image segmentation," SIAM J. Imaging Sciences, vol. 6, no. 3, pp. 1274-1317, 2013, arXiv preprint arXiv:1305.1206.

[5] Christian Ronse, "Partial partitions, partial connections and connective segmentation," Journal of Mathematical Imaging and Vision, vol. 32, no. 2, pp. 97-125, 2008.

[6] Christian Ronse, "Adjunctions on the lattices of partitions and of partial partitions," Applicable Algebra in Engineering, Communication and Computing, vol. 21, no. 5, pp. 343-396, 2010.

[7] Laurent Guigues, Jean Pierre Cocquerez, and Hervé Le Men, "Scale-sets image analysis," International Journal of Computer Vision, vol. 68, no. 3, pp. 289-317, 2006.

[8] F. Meyer and S. Beucher, "Morphological segmentation," Journal of Visual Communication and Image Representation, vol. 1, no. 1, pp. 21-46, Sept. 1990.

[9] Bangalore Ravi Kiran, Jean Serra, et al., "Global-local optimizations on hierarchies of segmentations," Pattern Recognition, vol. 47, no. 1, pp. 12-24, 2014.

[10] Philippe Salembier and Luis Garrido, "Binary partition tree as an efficient representation for image processing, segmentation, and information retrieval," IEEE Transactions on Image Processing, vol. 9, no. 4, pp. 561-576, 2000 .

[11] Fernand Meyer and Laurent Najman, "Segmentation, minimum spanning tree and hierarchies," in Mathematical morphology: from theory to applications, L. Najman and H. Talbot, Eds., pp. 229-261. ISTE / J. Wiley \& Sons, 2010

[12] Jean Cousty, Laurent Najman, and Jean Serra, "Raising in watershed lattices," in Image Processing, 2008. ICIP 2008. 15th IEEE International Conference on. IEEE, 2008, pp. 2196-2199.
[13] Jean Serra, Corinne Vachier, and Fernand Meyer, "Levelings," in Mathematical Morphology: From Theory to Applications, pp. 199-228. Wiley Online Library, 2013.

[14] Cédric Allène, Jean-Yves Audibert, Michel Couprie, and Renaud Keriven, "Some links between extremum spanning forests, watersheds and min-cuts," Image and Vision Computing, vol. 28, no. 10, pp. 1460-1471, 2010.

[15] Philippe Salembier, Albert Oliveras, and Luis Garrido, "Antiextensive connected operators for image and sequence processing," IEEE Transactions on Image Processing, vol. 7, no. 4, pp. 555-570, 1998.

[16] Laurent Najman, "On the equivalence between hierarchical segmentations and ultrametric watersheds," Journal of Mathematical Imaging and Vision, vol. 40, no. 3, pp. 231-247, 2011.

[17] Jean Serra, "A lattice approach to image segmentation," Journal of Mathematical Imaging and Vision, vol. 24, no. 1, pp. 83-130, 2006.

[18] Jean Cousty, Laurent Najman, and Benjamin Perret, "Constructive links between some morphological hierarchies on edge-weighted graphs," in Mathematical Morphology and Its Applications to Signal and Image Processing, pp. 86-97. Springer, 2013.

[19] Pierre Soille, "Constrained connectivity for hierarchical image partitioning and simplification," Pattern Analysis and Machine Intelligence, IEEE Transactions on, vol. 30, no. 7, pp. 1132-1145, 2008.

[20] Pierre Soille and Jacopo Grazzini, "Constrained connectivity and transition regions," in Mathematical Morphology and Its Application to Signal and Image Processing, pp. 59-69. Springer, 2009.

[21] L. Najman and M. Schmitt, "Geodesic saliency of watershed contours and hierarchical segmentation," IEEE Transactions on Pattern Analysis and Machine Intelligence, vol. 18, no. 12, pp. 1163-1173, December 1996. 